\section{Predicting behavior}

$\mathbf{P}$ sychopaths who have been convicted of murder are able to associate violent and pleasant thoughts with greater ease than other psychopaths or non-psychopathic murderers, concludes a recent study in Nature ${ }^{1}$. The paper describes a modified version of the Implicit Association Test (IAT), used previously to identify 'hidden' attitudes toward race, gender, weight and sexual preference. The authors suggest the test could help distinguish psychopaths "who are likely to commit extremely violent offences from those who are not." However, it seems unlikely that this test can be used to reliably predict individual behavior, and researchers need to consider the potential consequences of leading the public to believe that such selection is possible.

The current standard for identifying psychopaths is the Psychopathy Checklist-Revised (PCL-R), originally designed in 1991 by Robert Hare at the University of British Columbia. The test uses interviews and detailed case histories to rank individuals on a set of criteria that define a 'prototypical' psychopath. People who score more than 30 of a possible 40 points are considered psychopathic, and the PCL-R score is significantly correlated with violent behavior ${ }^{2}$.

The IAT, first described by Anthony Greenwald and colleagues in 1998 , is a relatively simple test that requires less training to administer. In the version used in the Nature study, subjects pressed one of two buttons to categorize a series of uppercase words as 'pleasant' or 'unpleasant' and lowercase words as 'violent' or 'peaceful'. Normal subjects are quick to respond when the same key is assigned to both 'violent' and 'unpleasant', concepts normally associated with each other. However, if 'violent' and 'pleasant' words are assigned to the same key, subjects are often momentarily conflicted, which delays their responses. This lag is inversely correlated with how strongly people associate the concepts. (For details and demonstration versions of the IAT, see http://implicit.harvard.edu/.) In the Nature study ${ }^{1}$, psychopathic murderers responded more quickly than non-psychopathic murderers or psychopathic non-murderers during the violent-pleasant word association condition.

A major strength of the IAT is that it measures beliefs that individuals are not always willing or able to admit, even to themselves, and it is difficult to fake. For example, in one study white participants were unable to show a lack of preference for white faces when they were instructed to do so, and in another study heterosexual participants were unable to fake positive attitudes toward homosexuality ${ }^{3}$. The test may therefore be particularly advantageous for use with psychopaths, who are often adept at lying and feigning emotions.

However, neither of these tests reliably predicts individual behavior. Although high scorers on the PCL-R are 3-4 times more likely than non-psychopaths to commit a violent offense after release from prison ${ }^{2}$, the test only measures the statistical likelihood of violence over a population. Furthermore, IAT results can differ depending on test context. Thus, participants who usually show a preference for white faces when taking the race version of the IAT may show a reduced preference if they view images of admired black faces and disliked white faces immediately before the test ${ }^{4}$. Such context sensitivity reduces the test's usefulness in measuring long-term behavioral tendencies.

More accurate prediction of psychopathic behavior may result from a better understanding of its underlying neural basis. Brain imaging and lesion data suggest that psychopaths have abnormalities in the amygdala and orbitofrontal cortex ${ }^{5}$. These areas are implicated in the ability to associate a particular behavior with a negative or rewarding outcome-processes that seem to be disrupted in psychopaths. However, it is not clear what causes such anatomical defects or whether they directly contribute to the observed behavioral problems. Thus attempts to use brain imaging to predict violent behavior are unlikely to find wide forensic application in the foreseeable future.

The ultimate goals of forensic psychology are to assess the risks of future violence in order to protect the public and to provide proper treatment of violent offenders. In many US states, sexually violent offenders can be forced to undergo further treatment via civil commitment after they have served their prison time; the PCL-R is used in making such decisions. For this reason, Hare and colleagues have gone to great lengths to provide formal training programs for the proper use of the PCL-R (www.hare.org). The IAT has not yet been used in the courtroom, and its designers do not recommend this test for individual selection, only for the evaluation of group tendencies.

In his short story, The Minority Report, science fiction writer Philip K. Dick speculates on the complications of a future in which the police use psychic 'precogs' to detect violent crimes before they happen. Despite such cautionary tales, however, the public desire for scientific tests of behavioral tendencies may not be easy to ignore-and not just for distinguishing psychopaths. A number of assessment tests are already in use for screening potential employees, and the IAT may not be far behind. It has been used for purposes ranging from the trivial (testing consumer preferences) to those with considerable potential for abuse, such as testing racial biases. As our knowledge of the neurobiological basis of behavior increases, the accuracy and usefulness of behavioral assessment tools will undoubtedly improve, but scientists must take the lead in promoting their appropriate application.

1. Gray, N.S., MacCulloch, M.J., Smith J., Morris, M. \& Snowden, R.J. Nature 423, 497-498 (2003).

2. Hare, R.D. in Criminal Justice, Mental Health, and the Politics of Risk (eds. Gray, N, Laing, J. \& Noaks, L.) 27-47 (Cavendish Publishing, London, 2002).

3. Greenwald, A.G. \& Nosek, B.A. Z. Exp. Psychol. 48, 85-93 (2001).

4. Dasgupta, N. \& Greenwald, A.G. J. Pers. Soc. Psychol. 81, 800-814 (2001).

5. Blair, R.J.R. Br. J. Psychiatry 182, 5-7 (2003). 